\title{
Deep Neural Network Learning for Detection and Grading of Diabetic Retinopathy
}

\author{
Akshay Jakkidi Reddy ${ }^{1}$, James B. Martel ${ }^{2 *}$ \\ ${ }^{1}$ Department of Ophthalmology, California Northstate University, Rancho Cordova, USA \\ ${ }^{2}$ Department of Graduate Medical Education, California Northstate University, Elk Grove, USA
}

*Corresponding author: James B. Martel, Department of Graduate Medical Education, California Northstate University, Elk Grove, United States of America; E-Mail: James.Martel@cnsu.edu

\section{ABSTRACT}

Purpose. To automate the retinal screening process and to improve chances of early detection/diagnosis of diabetic retinopathy which may reduce vision impairment cases due to the disease

Methods. Experiments were conducted on EYEPACS dataset that had retinal fundus images of patients with diabetic retinopathy in all severity levels. Based on the learning done by the Inception networks during the training phase, the images were classified to 5 classes based on the severity as: Normal, Mild, Moderate, severe and proliferative diabetic retinopathy. The images were augmented to increase the number of images with the disease and were randomly chosen for the training, validation and test datasets.

Results: The training was conducted using the Inception network for 100 epochs and validation accuracy was tested every 1000 iterations on a total of 109,540 images. The major misclassifications that occurred were between the adjacent classes of severity. It achieved a sensitivity of $94.6 \%$, specificity of $81.4 \%$, accuracy of $92 \%$ and precision of $93.2 \%$.

Conclusion: The retinal fundus images were examined and automated using Inception network by training the images from scratch. This model can be applied for detection of other retinal diseases, as the model weights are learnt to recognize subtle features present in the retinal images.

\section{INTRODUCTION}

Deep Neural Network (DNN) has provided an automated decision-making platform that allows computers to learn from past experience and make decisions on novel tasks. The goal is to train a computer to learn in a similar fashion to a human, but analyze information with the meticulous nature of a computer. Applying a deep neural network to analyze retinal images would assist in the early detection of diseases that progressively lead to vision loss such as diabetic retinopathy. DNN is known for its ability to learn complex patterns/features and extract knowledge from data. It facilitates deeper understanding, classification on various extracted features. Applying this technique to retinal fundus images, facilitates automation of disease diagnosis as well as monitoring of disease progression. Timely diagnosis and treatment is imperative for protection of vision.
Classification of diabetic retinopathy severity is a timeconsuming task as it involves analyzing several features of a fundus image which are often subtle and easily overlooked. Automated detection techniques using a 'learnt' model can be used to aid physicians in diagnosis and expedite treatment. Previous studies have demonstrated on disease classification using the retinal blood vessel data. [1-3]. The input images undergo a pre-processing step where noise, optic disk identification is carried out and a few papers to extract the retinal blood vessel. The area of the blood vessels is used for the detection of diabetic retinopathy. Abnormalities in the image are identified and the identified features are used to group the images according to the disease severity level. Most of the machine learning techniques are a two-class problem that uses K-nearest neighbor algorithm and support vector machine to do the classification [4]. 
The algorithm uses Fuzzy $\mathrm{C}$ means to allow features of the images to belongs to multiple clusters of information. The features that are extracted are given to support use within vector machine classifier to group the features as blood vessels, microaneurysms, exudates and texture features [5]. In a research where an automation technique which uses neural network to classify the diseased images as normal, non-proliferative diabetic retinopathy, proliferative diabetic retinopathy, the method uses morphological operations and image processing techniques in addition to texture analysis methods to extract the features for the neural network. Machine learning algorithms like support vector machine was used for a five-class classification problem where the images with diabetic retinopathy were classified to five different classes based on the severity level of the disease in the retinal image. The sensitivity and specificity that it recorded were $82 \%$ and $86 \%$ respectively [6].

Previous studies have focused on addressing specific characteristics of DR, such as microaneurysms or vessel segmentation, in order to generate a diagnosis. DNN is an artificial neural network that uses an algorithm to train the network on the desired functionality. The concept is modelled after human brain, solely for decision making with respect to a recognition/classification problems. DNN can be considered as a representational learning method which uses multiple data processing layers to learn and generate a model to represent the data in the dataset [7]. It has shown reliable and promising results with most of the computer vision problems. Convolutional neural network (CNN) is a deep neural network where convolution filters are moved across the image, detecting edge features present in the form of a line denoting a straight edge or a circle which could be a lesion/polyp at various orientations and locations. This is followed by detecting the arrangement of these edge features leading to the parts of the feature of interest and subsequent layers enables the classification of the feature of interest by combining the information retrieved from the previous layer. The main characteristics of $\mathrm{CNN}$ is in its increased number of layers. With more layers, there is increased abstraction and feature extraction. The filters are updated as they learn from the training data. These algorithms are parallelizable in nature and their results are probabilities indicating their chances for the input to belong to a particular class or class label [25]. Supervised learning is a machine learning technique which uses labeled data that guides the network towards learning the data. Supervised learning uses images that are labeled to its respective category of classes [8].

Convolutional neural network is used to detect the presence of exudates in the input image. Pre-processed image is then given to the model to extract the exudates candidates and classify them. Retinal blood vessel segmentation was done for disease identification purpose. There was no pre-processing step done to the input images. They used a 10-layer architecture classifying the pixel as vessel or non-vessel. In a few researches the input retinal images are patched to size $27 \times 27$. The image patches are then given as input to the layers of convolutions, pooling and fully connected network model for classification of pixels to either vessel or non-vessel classes. The binary classification problems use the deep network architecture for individual detection of microaneurysms and exudates [9]. The proposed technique was applied on $65 \times 65$ patches and are modelled to locate only one abnormality. They use an eight-layer network of convolution, max pooling and fully connected layer.

Fine-grained discriminant features are learnt using multiple filter sizes in architecture models like GoogleNet, VGGNet and Residual network(ResNet). GoogleNet is a 22 layer architecture which improved the efficiency of utilizing the computing resources while keeping the computational cost to a lower value. VGGNet is a 16-layer architecture built using $3 \times 3$ convolutions and $2 \times 2$ pooling layers. The model proved the importance of a deep network beneficial for higher classification accuracy. The input to the network inspired from the above 3 networks used a hybrid color space input image LGI, where L represents the luminance channel, $G$ is the green channel and $I$ is the intensity channel. These were derived from $L^{*} a * b, R G B, I 1, I 2$, I3 color spaces; taking into consideration only those channels that provided the most chrominance and/or luminance in the respective color space of the retinal image [10]. The $\mathrm{L}$ channel provides values in the range 0-100 while the other two channels have intensity values in the range 0-255. Green channel in RGB color space has most of the retinal information that is required for classification [11]. The models that were inspired from GoogleNet is called as combined kernel with multiple losses (CKML). The model which was inspired from VGGNet and ResNet is VGGNet with extra kernel (VNXK) [12].

Inception network, state of the art for detection and classification purposes used for diabetic retinopathy severity classification. Grading the severity level of a fundus image involves multiple factors and it can also differ with doctors and their perspective. The datasets used in this work was labelled and cross validated for consistency between 54 Ophthalmologists. Around 7 reads were considered to finalize the labels that were used for training the network. Their research assures a clean dataset with reliable images 
labelled by Ophthalmologists. The Inception was originally a 1000 class classification problem. The last layer was modified to use it for the 5 class classification problem [13].

\section{METHOD AND STRUCTURE}

Training of the algorithm was performed done using the EyePACS-1 dataset. EyePACS is comprised of 9963 images from 4997 individuals. these images were graded by 8 ophthalmologists with a redundancy of 8 grades per image. Severity was graded according to the international clinical diabetic retinopathy disease severity scale. This image set contains predominantly disease-free images, given a Class 0 designation. Diseased images were classified by order of increasing severity with mild, moderate and severe nonproliferative DR and proliferative DR given a class of 2-4 respectively. There is a greater number of normal's than any other class. This caused there to be a skew in the algorithm that biased selection of Class 0 . Figure 1 shows the dataset split [14]. To balance this bias, 10 augmentation techniques were applied to the images in the dataset. The augmentations applied were random combinations of rotation, flipping, adding speckle noises and rotating/flipping a noised image. Out of these new variants, a total of 22000 images were considered per class at the time of training [15].

The input image pixels were taken into an array and saved in an uncompressed format .npz In this 5 class problem, the one hot vector format of labels had a shape of the number of the images in the dataset and the number of classes. The images are shuffled and a batch of 50 images are given as input to the network. This is done so that the network doesn't learn in the same format/ pattern [16].

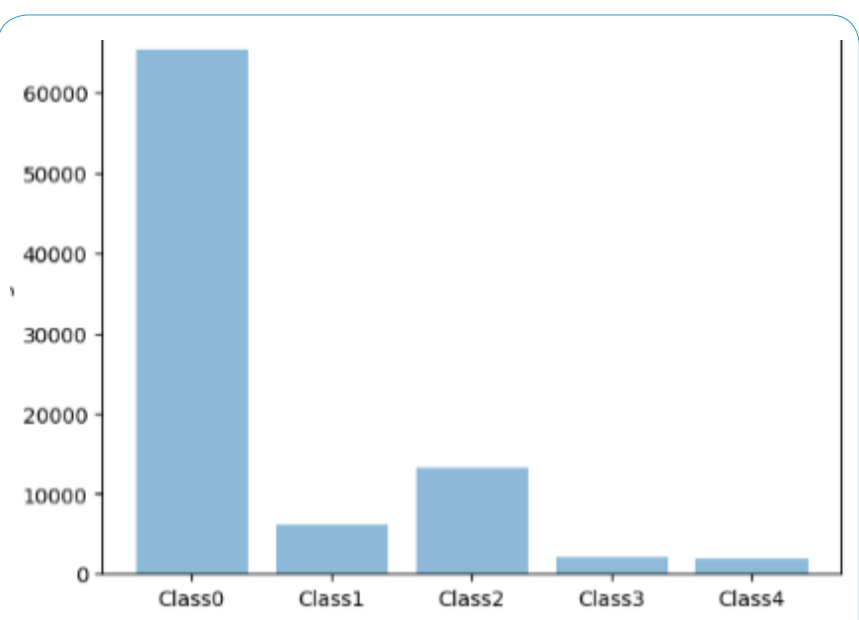

Figure 1: Dataset split.

\section{NETWORK MODEL}

Inception networks applies the Network-in-network concept, where $1 \times 1$ convolutions are applied so that the features extracted from convolutional layers can be combined towards better results. It consists of convolutional, batch normalizations, max/average pooling, dropout, fully connected layers and Softmax at the output layer. In a convolutional layer, the computations are carried out by a set of learnable filters. It takes the inner product of the filter which contains random values if training from scratch or learned values if a pre-trained model is used, with a receptive field and applies an activation function for every section in the input image. The resulting values obtained for one filter is put together to form a feature map. In a forward pass, the convolution filters convolve across the input image and their respective output for every spatial location gets recorded for the succeeding layers. The layer carries out convolution both spatially and also to the full depth at the input layer. The error calculated at the end of every feed forward pass is used to update the weights, generating new convolution filters which are learnt from its prior forward pass. The max pooling layer gets the most significant features from a given set of values. It is used to reduce parameters and computations involved within a network. There are 2 types of pooling operation: Max pooling which gets the maximum value from the window under consideration. Average pooling deals with the average of the values within the window size. The dropout layer carries out a model averaging by dropping a random set of units from the network while training [17]. It is a regularization technique which is applied to reduce overfitting in a network. Dropout removes both the input and its corresponding output values based on a probability. The Inception network used a drop out ratio of $40 \%$ [23] before the Softmax classification layer [18].

All the convolutional layers in Inception network uses Rectified Linear Unit (ReLU) activation function. ReLU is a non-linear activation function that employs a function whose is threshold at zero. This is an activation function which enables faster learning, speeds up the network training and it promotes faster convergence of stochastic gradient descent when compared to other activation functions. A total of 27 layers are used for training and testing the network for severity level disease classification [19].

The Inception network utilizes the computations as efficiently as possible by selecting optimal sizes of filters for convolutions and by using aggressive regularizations. The outputs from $1 \times 1,3 \times 3$ and $5 \times 5$ filters were concatenated 


\section{Research Article}

to form the input features for the succeeding layer. The Inception network uses series of Inception modules comprising of concatenated outputs of multiple filter sizes. The smaller filter sizes used in the network are chosen in such that they do not lead to expensive computations. The $1 \mathrm{x} 1$ convolutions are carried out initially to reduce the computations that the succeeding filters of sizes $3 \times 3$ and $5 \times 5$ convolutions would require to do. The module layers are built by stacking up convolutional layers of varied filter sizes, and applying max-pooling to reduce the output sizes. The modules are then concatenated to a single output vector forming input to the next stages in the network. The final classification layer uses Softmax classifier with 5 units indicating the 5 classes to which the images are to be classified. In this paper, diabetic retinopathy disease severity classification was carried out using transfer learning process using a pre-trained Inception model and also by training an Inception network model from scratch on the dataset of balanced images [20].

\section{TRAINING}

Instead of training an Inception network from scratch, the saved model with the existing weights, can be used to check how the network recognizes/respond to the images in the new dataset. Transfer learning is a technique where the parameters saved as a result of the training/learning procedure are used to learn similar types of problem. The last layer in the network is modified to classify the new dataset to the desired number of classes. The saved model weights are constant throughout the transfer learning process. The parameters for the last layer alone go through the learning process for the set number of epochs.

Inception was originally trained on a dataset of random objects. The network records a top-5 error of $6.67 \%$ with the ImageNet dataset with receptive field sizes of $299 \times 299$. The Inception model weights were trained on random objects in the ImageNet dataset. This dataset is a very large-scale image dataset. A few example categories which comprises this dataset includes: images of terrestrial animals, aquatic/ marine organisms, objects, trees, cars, food, person and structures. From the experiments tabulated in table1 we can see that it was not able to carry out the severity classification of the retinal images. The severity differences between the retinal images from class0, class 1 , class 2 are very subtle. It required intense learning using a wide and deep network like Inception from scratch for the network to identify the image features that can be used to categorize them to the respective classes. Subsequent retaining took place [21].

The network model was trained from scratch using the images in the balanced training dataset. Every convolution layer in the network model used a square filter of optimal size across every input image channel.

This deep neural network technique extracts the feature vector from the raw input image pixel and classifies them to either of the groups using fully connected layers. The values obtained from a single input image as a result of convolution is summed up for the whole input map and then the result is given to an activation function. Unlike in sigmoid activation function, in ReLU the gradients do not become 0

\begin{tabular}{|c|c|}
\hline \multicolumn{2}{|c|}{ Table 1: Inception transfer learning parameters } \\
\hline Transfer Learning Parameters & Values \\
\hline Cost Estimation & Softmax cross entropy with logits \\
\hline Optimizer & Gradient Descent Optimizer \\
\hline Number of Epochs & 50,000 \\
\hline Images per class & 22000 \\
\hline Training data & $70 \%$ \\
\hline Validation data & $20 \%$ \\
\hline Test data & $10 \%$ \\
\hline
\end{tabular}

\begin{tabular}{|c|c|c|c|}
\hline \multicolumn{4}{|c|}{ Table 2: Inception retraining results } \\
\hline Learning Rate & Training Accuracy & Validation Accuracy & Test Accuracy \\
\hline 1.5 & $47.0 \%$ & $44.0 \%$ & $43.50 \%$ \\
\hline 0.1 & $45.0 \%$ & $34.0 \%$ & $36.3 \%$ \\
\hline 0.01 & $55.5 \%$ & $47.7 \%$ & $50.8 \%$ \\
\hline 0.001 & $50.8 \%$ & $46.9 \%$ & $48.20 \%$ \\
\hline 0.005 & $56.2 \%$ & $46.1 \%$ & $50.4 \%$ \\
\hline
\end{tabular}


after a few steps in the training process. The pooling layer retains the pixels that has the most significance in the area under consideration. The pooling used in this paper is $2 \times 2$ max pooling with a stride of 2 . The number of pixels with which we move across the image is called as stride [22]. The resulting volume size can be calculated using the input size, filter size and the stride value. These layers are independent of the number of channels in the input image. The max pooling operation does not do any form of training, instead it fetches us with the most promising features in the image [23].

The gradient optimizer used is gradient descent optimizer with momentum. A learning rate of 0.001 and the cost was estimated using categorical cross entropy. The gradient descent algorithm is used to minimize the cost which is in terms of weights and bias. Categorical cross entropy is computed between the predictions and the targets. It indicates how close the predicted result is to the target label. In the final output layer, 5 neurons: 0 indicating the non-diseased class and 1 indicating the mild diseased class, 2 indicates the moderate diseased class, 3 severe NPDR and 4 indicates PDR. The training was initially done on a smaller dataset, with 5000 images in every class. Due to the wide and deep nature of the network, it was found to be overfitting towards the latter half of the training. Applying augmentations to the images, the number of images in the dataset were increased. Training the Inception from scratch using the bigger dataset showed improved training, validation and test accuracy values [24].

This research problem focused on learning multi-level features extracted from huge dataset of images. The experiments conducted involved usage of deep and wide network layers for predictive classification/decision making. Due to larger number of input images in the training dataset in its training phase, it involved computations in a larger scale and generation of numerous parameters like bias values, weight matrices from one neuron to the other, for all the feature maps in every layer of the network model. GPU nodes that can carry out the computationally intensive tasks were used in the training phase and also in the testing phase. The network took around 2 weeks of NVIDIA's Tesla P-100 GPU hours to complete the training phase. The GPU node used Pascal architecture and it used CUDA 8.0 toolkit for GPU learning [25].

\section{RESULTS}

The Inception network was applied to the dataset to get the following results. Training the Inception network model from scratch showed steady learning on the images in the dataset. The validation accuracy was found every

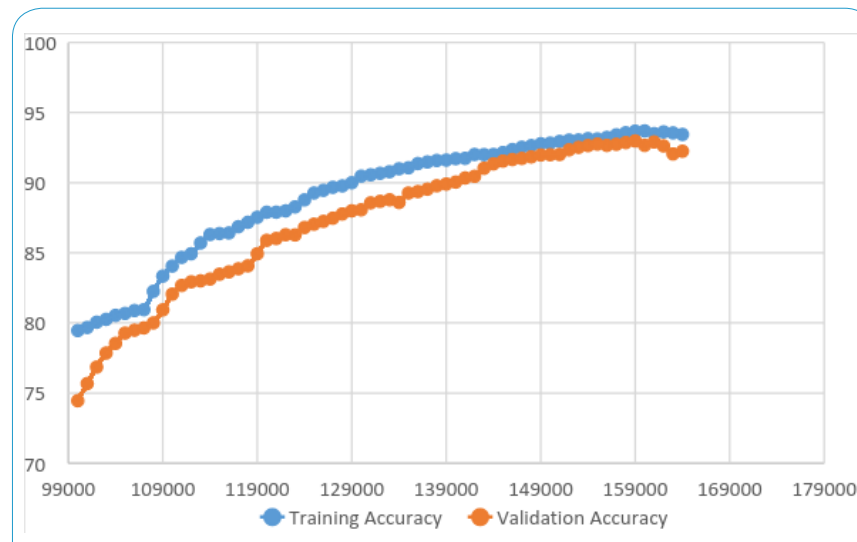

Figure 2: Accuracy Plot

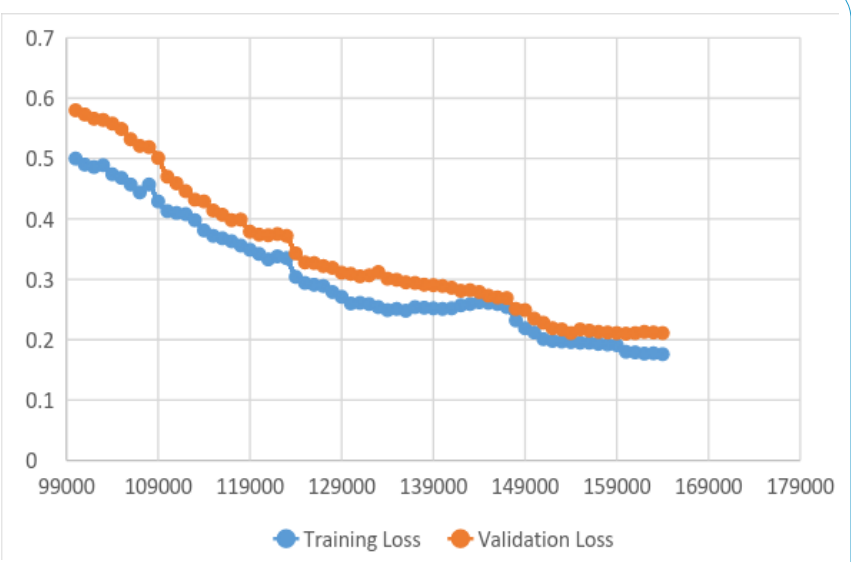

Figure 3: Category loss plot

1000 iterations using the learnt model at that respective iteration. The model showed significant learning and it gave training and validation accuracy values of $93 \%$ and $94 \%$ respectively. Figure 3 shows the accuracy plot and Figure 4 shows the plot for the loss at the respective iteration.

The 4 main parameters that are required for finding the performance measurements were calculated. The number of correctly detected diabetic retinopathy images is True Positive. True Negative is the number of images that were correctly classified as non-diabetic retinopathy images. False positive indicates the number of normal images that were wrongly classified as diseased and False Negative images is the number of diseased images that were correctly classified as diabetic retinopathy images. These 4 parameters were used to calculate sensitivity which indicates the chances for the test to be positive for images that shows signs of the disease. Specificity measures the chances for a normal image to be classified as normal itself. These performance measurements are calculated as follows: 
Research Article

$$
\begin{array}{ll}
\text { - } & \text { Sensitivity }=\frac{T P}{T P+F N} \\
\text { - } & \text { Specificity }=\frac{T N}{T N+F P} \\
\text { - } & \text { Accuracy }=\frac{T P+T N}{T P+T N+F P+F N} \\
\text { Precision } & =\frac{T P}{T P+F P}
\end{array}
$$

\begin{tabular}{|l|l|l|l|l|l|}
\hline Parameters & Value & Sensitivity & Specificity & Accuracy & Precision \\
\hline Learning Rate & 0.001 & & & & \\
\cline { 1 - 2 } Number of Epoch & 100 & & & & \\
\cline { 1 - 2 } Training images & 78518 & \multirow{2}{*}{$94.6 \%$} & \multirow{2}{*}{$81.8 \%$} & $91.3 \%$ & \\
\cline { 1 - 2 } Validation images & 19630 & & & & \\
\cline { 1 - 2 } Test Image & 300 & & & & \\
\cline { 1 - 2 } & 50 & & & & \\
\hline
\end{tabular}

\section{DISCUSSION}

Deep Learning was applied to the retinal images for classification based on diabetic retinopathy disease severity level as based on the international standard. Inception networks for machine learning can be trained via saved state and through de novo. It appears as though the differences between DR severity classes is too minor to be picked up using a premade algorithm. In order to pick up the subtle differences, using the inception network, de novo algorithm generation must be done. The inception network was trained purely from the EyePacs dataset to classify the images according to the severity of the abnormalities present in the retinal fundus images. The accuracy values can be improved if the dataset can be analyzed and filtered from images that are non-gradable by medical professionals. This model can be applied for detection of other retinal diseases, as the model weights are learnt to recognize subtle features present in the retinal images. Automating the retinal screening process can facilitate better and faster access to medical treatment/screening thereby reducing the chances for vision impairment/ timely treatment among the diseased.

\section{REFERENCES}

1. Mo J, Zhang L. Multi-level deep supervised networks for retinal vessel segmentation. Int J Comput Assist Radiol Surg. 2017 Dec;12(12):2181-2193. doi: 10.1007/s11548-017-1619-0. Epub 2017 Jun 2. PMID: 28577175.

2. Eftekhari N, Pourreza HR, Masoudi M, Ghiasi-Shirazi K, Saeedi E. Microaneurysm detection in fundus images using a two-step convolutional neural network. Biomed Eng Online. 2019 May 29;18(1):67. doi: 10.1186/s12938-019-0675-9. PMID: 31142335; PMCID: PMC6542103.

3. Hua CH, Huynh-The T, Lee S. Retinal Vessel Segmentation using Round-wise Features Aggregation on Bracket-shaped Convolutional Neural Networks. Annu Int Conf IEEE Eng Med Biol Soc. 2019 Jul;2019:36-39. doi: 10.1109/ EMBC.2019.8856552. PMID: 31945839.
4. Usman Akram M, Khalid S, Tariq A, Khan SA, Azam F. Detection and classification of retinal lesions for grading of diabetic retinopathy. Comput Biol Med. 2014 Feb;45:161-71. doi: 10.1016/j.compbiomed.2013.11.014. Epub 2013 Dec 1. PMID: 24480176 .

5. Prentašić P, Lončarić S. Detection of exudates in fundus photographs using deep neural networks and anatomical landmark detection fusion. Comput Methods Programs Biomed. 2016 Dec;137:281-292. doi: 10.1016/j.cmpb.2016.09.018. Epub 2016 Oct 6. PMID: 28110732.

6. Gulshan V, Peng L, Coram M, Stumpe MC, Wu D, Narayanaswamy A, Venugopalan S, Widner K, Madams T, Cuadros J, Kim R, Raman R, Nelson PC, Mega JL, Webster DR. Development and Validation of a Deep Learning Algorithm for Detection of Diabetic Retinopathy in Retinal Fundus Photographs. JAMA. 2016 Dec 13;316(22):2402-2410. doi: 10.1001/jama.2016.17216. PMID: 27898976

7. Ometto G, Erlandsen M, Hunter A, Bek T. The role of retinopathy distribution and other lesion types for the definition of examination intervals during screening for diabetic retinopathy. Acta Ophthalmol. 2017 Jun;95(4):400-404. doi: 10.1111/aos.13334. Epub 2016 Nov 19. PMID: 27864877.

8. Walter T, Klein JC, Massin P, Erginay A. A contribution of image processing to the diagnosis of diabetic retinopathy--detection of exudates in color fundus images of the human retina. IEEE Trans Med Imaging. 2002 Oct;21(10):1236-43. doi: 10.1109/ TMI.2002.806290. PMID: 12585705.

9. Sopharak A, Uyyanonvara B, Barman S, Williamson TH. Automatic detection of diabetic retinopathy exudates from nondilated retinal images using mathematical morphology methods. Comput Med Imaging Graph. 2008 Dec;32(8):720-7. doi: 10.1016/j.compmedimag.2008.08.009. Epub 2008 Oct 18. PMID: 18930631 .

10. Schlegl T, Waldstein SM, Bogunovic H, Endstraßer F, Sadeghipour A, Philip AM, Podkowinski D, Gerendas BS, Langs G, SchmidtErfurth U. Fully Automated Detection and Quantification of Macular Fluid in OCT Using Deep Learning. Ophthalmology. 2018 Apr;125(4):549-558. doi: 10.1016/j.ophtha.2017.10.031. Epub 2017 Dec 8. PMID: 29224926.

11. Sánchez CI, Niemeijer M, Išgum I, Dumitrescu A, SuttorpSchulten MS, Abràmoff MD, van Ginneken B. Contextual computer-aided detection: improving bright lesion detection in retinal images and coronary calcification identification in CT scans. Med Image Anal. 2012 Jan;16(1):50-62. doi: 10.1016/j. media.2011.05.004. Epub 2011 Jun 1. PMID: 21689964.

12. Liskowski P, Krawiec K. Segmenting Retinal Blood Vessels With Deep Neural Networks. IEEE Trans Med Imaging. 2016 Nov;35(11):2369-2380. doi: 10.1109/TMI.2016.2546227. Epub 2016 Mar 24. PMID: 27046869.

13. Eladawi N, Elmogy MM, Ghazal M, Helmy O, Aboelfetouh A, Riad A, Schaal S, El-Baz A. Classification of retinal diseases based on OCT Images. Front Biosci (Landmark Ed). 2018 Jan 1;23:247-264. doi: 10.2741/4589. PMID: 28930545.

14. Salamat N, Missen MMS, Rashid A. Diabetic retinopathy techniques in retinal images: A review. Artif Intell Med. 2019 Jun;97:168-188. doi: 10.1016/j.artmed.2018.10.009. Epub 2018 Nov 16. PMID: 30448367. 
15. Sarıül M, Ozyildirim BM, Avci M. Differential convolutional neural network. Neural Netw. 2019 Aug;116:279-287. doi: 10.1016/j. neunet.2019.04.025. Epub 2019 May 10. PMID: 31125914.

16. Harangi B, Toth J, Hajdu A. Fusion of Deep Convolutional Neural Networks for Microaneurysm Detection in Color Fundus Images. Annu Int Conf IEEE Eng Med Biol Soc. 2018 Jul;2018:37053708. doi: 10.1109/EMBC.2018.8513035. PMID: 30441176.

17. Hassan B, Hassan T, Li B, Ahmed R, Hassan O. Deep Ensemble Learning Based Objective Grading of Macular Edema by Extracting Clinically Significant Findings from Fused Retinal Imaging Modalities. Sensors (Basel). 2019 Jul 5;19(13):2970. doi: 10.3390/s19132970. PMID: 31284442; PMCID: PMC6651513.

18. Roychowdhury S, Koozekanani DD, Parhi KK. Automated detection of neovascularization for proliferative diabetic retinopathy screening. Annu Int Conf IEEE Eng Med Biol Soc. 2016 Aug;2016:1300-1303. doi: 10.1109/EMBC.2016.7590945. PMID: 28268564.

19. Nayak J, Bhat PS, Acharya R, Lim CM, Kagathi M. Automated identification of diabetic retinopathy stages using digital fundus images. J Med Syst. 2008 Apr;32(2):107-15. doi: 10.1007/ s10916-007-9113-9. PMID: 18461814.

20. Acharya UR, Lim CM, Ng EY, Chee C, Tamura T. Computerbased detection of diabetes retinopathy stages using digital fundus images. Proc Inst Mech Eng H. 2009 Jul;223(5):545-53. doi: 10.1243/09544119JEIM486. PMID: 19623908.
21. Ma J, Sheridan RP, Liaw A, Dahl GE, Svetnik V. Deep neural nets as a method for quantitative structure-activity relationships. J Chem Inf Model. 2015 Feb 23;55(2):263-74. doi: 10.1021/ ci500747n. Epub 2015 Feb 17. PMID: 25635324.

22. Lee H, Kwon H. Going Deeper With Contextual CNN for Hyperspectral Image Classification. IEEE Trans Image Process. 2017 Oct;26(10):4843-4855. doi: 10.1109/TIP.2017.2725580. Epub 2017 Jul 11. PMID: 28708555.

23. Shin HC, Roth HR, Gao M, Lu L, Xu Z, Nogues I, Yao J, Mollura D, Summers RM. Deep Convolutional Neural Networks for Computer-Aided Detection: CNN Architectures, Dataset Characteristics and Transfer Learning. IEEE Trans Med Imaging. 2016 May;35(5):1285-98. doi: 10.1109/TMI.2016.2528162. Epub 2016 Feb 11. PMID: 26886976; PMCID: PMC4890616.

24. Qin F, Gao N, Peng Y, Wu Z, Shen S, Grudtsin A. Finegrained leukocyte classification with deep residual learning for microscopic images. Comput Methods Programs Biomed. 2018 Aug;162:243-252. doi: 10.1016/j.cmpb.2018.05.024. Epub 2018 May 22. PMID: 29903491.

25. H. Greenspan, B. van Ginneken and R. M. Summers, "Guest Editorial Deep Learning in Medical Imaging: Overview and Future Promise of an Exciting New Technique," in IEEE Transactions on Medical Imaging, vol. 35, no. 5, pp. 1153-1159, May 2016, doi: 10.1109/TMI.2016.2553401. 